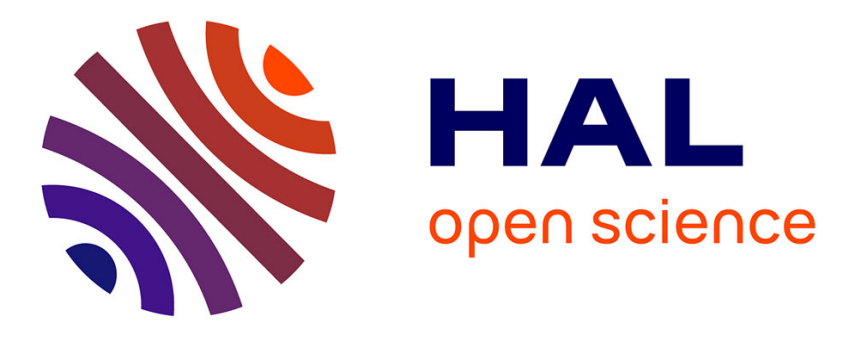

\title{
Les inscriptions arabes, coptes et guèzes des églises de Lālibalā
}

Wadi Awad Abullif, Emmanuel Fritsch, Claire Bosc-Tiessé, Marie-Laure Derat

\section{To cite this version:}

Wadi Awad Abullif, Emmanuel Fritsch, Claire Bosc-Tiessé, Marie-Laure Derat. Les inscriptions arabes, coptes et guèzes des églises de Lālibalā. Annales d'Éthiopie, 2010, 25 (1), pp.43-53. 10.3406/ethio.2010.1406 . halshs-02552669

\section{HAL Id: halshs-02552669 \\ https://shs.hal.science/halshs-02552669}

Submitted on 23 Apr 2020

HAL is a multi-disciplinary open access archive for the deposit and dissemination of scientific research documents, whether they are published or not. The documents may come from teaching and research institutions in France or abroad, or from public or private research centers.
L'archive ouverte pluridisciplinaire HAL, est destinée au dépôt et à la diffusion de documents scientifiques de niveau recherche, publiés ou non, émanant des établissements d'enseignement et de recherche français ou étrangers, des laboratoires publics ou privés. 


\title{
Les inscriptions arabes, coptes et guèzes des églises de Lālibalā Wadi Awad Abullif, Emmanuel Fritsch, Claire Bosc-Tiessé, Marie-Laure Derat
}

\section{Résumé}

Cet article publie et traduit des inscriptions arabes, coptes et guèzes relevées dans trois églises du site de Lālibalā : à Bēta Māryām, Bēta Giyorgis et Bēta Libānos. Pour la plupart, ce sont de simples ex-voto. Une attention particulière est accordée à l'une des inscriptions, figurant sur le pilier central de l'église de Bēta Māryām (l'āmda berhān) qui fait allusion à la construction de l'église.

\begin{abstract}
The Arabic, Coptic and Ge'ez inscriptions in Lālibalā In this article, we edit and translate Arabic, Coptic and Ge'ez inscriptions from three churches of the Lālibalā's site : Bēta Māryām, Bēta Giyorgis and Bēta Libānos. Most of them are simple ex-voto. A particular attention is given to one of these inscriptions, placed on the central pilar of the Bēta Māryām church ('āmda berhān) which refers to the carving of the church.
\end{abstract}

\section{Citer ce document / Cite this document :}

Awad Abullif Wadi, Fritsch Emmanuel, Bosc-Tiessé Claire, Derat Marie-Laure. Les inscriptions arabes, coptes et guèzes des églises de Lālibalā. In: Annales d'Ethiopie. Volume 25, année 2010. pp. 43-53;

doi : 10.3406/ethio.2010.1406

http://www.persee.fr/doc/ethio_0066-2127_2010_num_25_1_1406

Document généré le 08/03/2018 


\title{
Les inscriptions arabes, coptes et guèzes des églises de Lālibalā
}

\author{
Claire Bosc-Tiessé, Marie-Laure Derat, \\ Emmanuel Fritsch, Wadi Awad Abullif*
}

L'écriture de l'histoire des églises de Lālibalā concerne non seulement la fondation du site mais aussi son évolution sur la longue durée et exige la constitution d'un corpus documentaire aussi exhaustif que possible. Dans le registre de l'écrit, les inscriptions relevées à l'intérieur comme à l'extérieur - dans le cas unique de Bēta Māryām - des églises de Bēta Māryām, Bēta Giyorgis et Bēta Libānos viennent compléter une documentation très fragmentaire ${ }^{1}$.

Les inscriptions trouvées sur le site de Lālibalā sont de différentes natures. La plupart sont tracées à l'encre directement sur la roche. À Bēta Māryām en revanche, on trouve, d'une part, une inscription gravée dans le rocher sur le mur oriental de l'église, au-dessus d'une tombe qui vient se glisser sous le mur mais dont l'accès est extérieur; et d'autre part, une inscription écrit sur enduit sur le pilier central de l'église, que l'on appelle čamda berhān, "pilier de lumière». Qui plus est, ces inscriptions ne sont pas toujours dans la même langue: certes, beaucoup sont écrites en guèze mais certaines sont rédigées en arabe avec quelques mots de copte. Aucune de ces inscriptions ne comporte de date. Elles sont le plus souvent de simples ex-voto, signalant qu'un pèlerin venu d'Éthiopie ou d'Égypte s'est rendu sur les lieux, et laissant un souvenir de son passage. L'inscription sur l' ámda berhàn de Bēta Māryām, relève aussi d'un autre registre : c'est un "texte de fondation »" qui était donné à lire aux fidèles pénétrant dans l'église. Mais il faut employer le passé, puisque le pilier central de Bēta Māryām est désormais couvert d'un tissu qui dissimule l'inscription tout autant qu'il l'entoure d'une aura de mystère. Nous avons

\footnotetext{
C. B.-T., M.-L. D. \& E. F.: Centre Français des Études Éthiopiennes (USR 3137/UMIFRE 23), CNRS \& Ministère des Affaires Étrangères, Addis Abeba, Éthiopie ; W. A. A. : Franciscan Center for Oriental Christian Studies, Le Caire, Égypte.

1 Tous nos remerciements vont à Anaïs Wion qui nous a signalés les inscriptions guèzes de Bēta Giyorgis. Les investigations que nous avons ensuite menées nous ont permis de découvrir l'inscription arabe de cette église. Nous remercions aussi Robin Seignobos toujours prompt à rendre service et qui nous a aidés avec les re-transcriptions des caractères arabes.

2 Sur ce que nous entendons par « texte de fondation », voir infra.
} 


\section{Claire Bosc-Tiessé et al.}

choisi de présenter, traduire et donner des éléments d'interprétation pour les inscriptions arabes et coptes. Les ex-voto rédigés en guèze, moins précis, viendront seulement en contre-point de ce travail quand cela sera nécessaire. Il est possible que certaines inscriptions nous aient échappé, dans la mesure où celles qui ont été vues sont parfois très difficiles à lire. D'autres ne sont aujourd'hui plus visibles : ainsi, il y a quinze ans, Ugo Zanetti et Emmanuel Fritsch avaient-ils remarqué une inscription à Bēta Golgotā qui, depuis, est devenue si ténue qu'il nous a été impossible de la retrouver.

Ces inscriptions dénotent un usage : faire savoir qui est venu à Lālibalā ou qui a fait faire quelque chose en cet endroit. Alors que, la plupart du temps, ce type d'information figure en marge des manuscrits, sous la forme de donations, et n'est donc accessible qu'à ceux qui ont accès à ces manuscrits et peuvent les lire; ici l'information est divulguée au plus grand nombre puisqu'elle se trouve là où tout un chacun peut se rendre, et est visible dès que l'on entre dans l'église. Mais il y a une contradiction apparente entre la volonté de faire savoir par l'inscription et la langue employée dans celle-ci, puisque celles que nous présentons ici sont écrites en arabe avec parfois quelques mots de copte. Ce qui soulève la question des destinataires réels. Certes, il y a bien des lettrés éthiopiens qui connaissent l'arabe et qui peuvent servir de passeur entre les deux mondes. Mais ne faut-il pas plus sûrement penser que l'inscription n'est en quelque sorte qu'un aide mémoire, une image qu'il n'est pas nécessaire de lire mais dont la présence suffit à témoigner visuellement de ce qu'elle signifie ou rappelle. Elle fonctionnerait donc davantage comme un sceau que comme une inscription.

\section{Les inscriptions ex-voto de Bēta Libānos et de Bēta Giyorgis}

Dans les églises de Bēta Libānos et de Bēta Giyorgis, plusieurs inscriptions en guèze se lisent encore sur les piliers ainsi que, dans chacune de ces églises, une inscription en arabe. Ainsi, à Bēta Libānos, il y a encore trois inscriptions en guèze, deux sur des piliers dans la nef de l'église, en hauteur, et une sur le montant gauche de l'arche triomphale. Dans ce dernier cas, elle se trouve sous l'inscription arabe. Celle-ci se lit ainsi :

$$
\text { ادكر يا رب عبدك الخاطي ابو اسكندر؟ }
$$

«Souviens-toi, Seigneur, de ton serviteur, le pécheur, Abou Iskandar ${ }^{3}$ »

On peut se demander si le texte guèze inscrit au dessous en est une traduction. Il n'est pas totalement lisible. Écrit sur quatre lignes, seules les deux premières peuvent être déchiffrées :

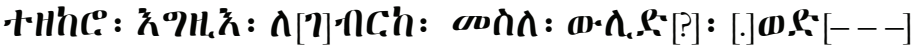

«Seigneur souviens-toi de ton serviteur avec $[---]$ »

Il ne s'agit donc pas d'une traduction terme à terme de l'inscription arabe. Mais, tout comme pour cette dernière, son auteur ou son commanditaire a choisi de faire un ex-voto en ces lieux.

3 La lecture d'Iskandar est douteuse. 
D'autres inscriptions en guèze, apparemment plus récentes d'après la graphie, ont été écrites sur d'autres piliers de l'église de Bēta Libānos.

De même à Bēta Giyorgis, on trouve quatre inscriptions en guèze et une en arabe sur les piliers latéraux, gauche et droit, de l'arche triomphale. Deux en guèze côté gauche ainsi qu'une troisième à droite de l'arche sont écrites en gros caractères dans une graphie antérieure au XVe siècle. Elles sont difficiles à déchiffrer mais les quelques mots qui se distinguent semblent bien pointer des prières ex-voto.

En les numérotant de gauche à droite, ce qu'on peut lire sur la première atteste

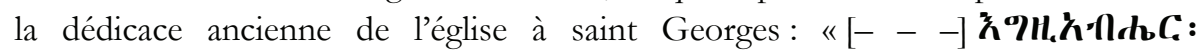

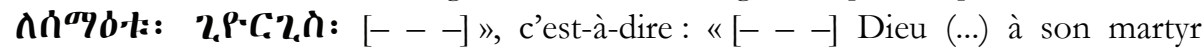

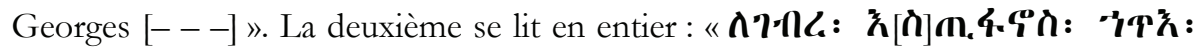

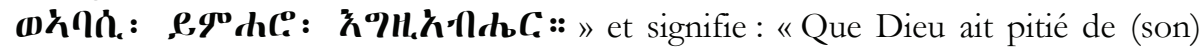
serviteur Esțifānos, pécheur et fautif ». Sur la troisième, à droite de l'arche qui mène au sanctuaire, nous ne pouvons distinguer que le mot W'ThC: qui indique qu'il s'agit d'une commémoration.

Plus à droite encore, sur le deuxième pilier engagé se trouvent une inscription en arabe et en-dessous une inscription en guèze, illisible. L'arabe en revanche se déchiffre bien :

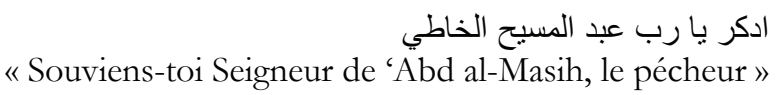

Le nom de 'Abd al-Masih, Serviteur du Messie, peut être rapproché du nom qui apparaît sur l' āmda berhān de Bēta Māryām - Serviteur du Christ, le premier étant en arabe, le second en copte. Il pourrait, sous toutes réserves, s'agir de la même personne, ce qui ne serait pas sans implication sur la contemporanéité des deux églises. Le fait que ce nom soit si courant nous empêche bien sûr de pousser l'hypothèse.

Posons une question qui peut être en rapport: cette inscription est placée bien en vue sur le pilier à l'entrée du sanctuaire : l'inscription a nécessairement été faite au vu et au su de tout le monde, ce qui plaide pour l'idée qu'elle a été faite pour ou par un personnage important.

Les inscriptions en guèze sont placées autour de l'inscription en arabe et on peut se demander si ce n'est pas celle-ci qui a donné l'idée, dans un deuxième temps, d'écrire celles en guèze.

\section{L'inscription arabe et copte de Bēta Māryām}

L'āmda berbān de l'église de Bēta Māryām occupe une position très particulière : situé en plein centre de la nef, il s'impose aux yeux de tous comme l'élément essentiel de cette église, sans qu'aucune nécessité architectonique ne puisse a priori expliquer sa place. Il a donc été réservé dans le rocher pour une raison précise qui nous échappait jusqu'à présent, mais dont beaucoup soupçonnaient que des 
éléments de réponse étaient à chercher derrière le voile qui le recouvre entièrement, du sol au plafond ${ }^{4}$.

Il semble que le pilier était déjà voilé dès le début du XVI ${ }^{\mathrm{e}}$ siècle quand le chapelain de l'ambassade du roi du Portugal auprès du roi d'Éthiopie, Francisco Alvares, visita le site peu après 1520. Sinon, comment comprendre la description qu'il donne de l'église de Bēta Māryām :

«L'église de notre Dame n'est pas si grande, que celle de Saint Sauveur, mais elle est beaucoup plus industrieusement labourée et d'un ouvrage plus artificiel, ayant trois nefs : dont celle du milieu est très haute, embellie de plusieurs tailles de roses, merveilleusement bien tirées sur la pierre même. Chacune d'icelles nefs a cinq colones, soutenans leurs arcs en voute, fort bien liées et y a une fort haute colone de surcroit vers la croisée, sur laquele sapuye un poile $»^{5}$.

Il faut ici comprendre «voile», et non «poile». Certes, il n'est pas certain que le pilier était entièrement voilé, mais on peut penser que si Francisco Alvares avait vu les inscriptions figurant sur celui-ci, il n'aurait pas manqué d'en parler. Nous ne disposons pas d'autres témoignages anciens concernant ce pilier.

Il faut ensuite attendre le XIX ${ }^{\mathrm{e}}$ siècle pour que des voyageurs s'arrêtent à Lālibalā et en fournissent une description détaillée. Mais rien n'est dit au sujet de l'camda berhān dans l'ouvrage de Gabriel Simon, alors qu'il décrit très précisément certains aspects de la décoration intérieure de Bēta Māryāmº ${ }^{6}$ De même si Augusto Monti della Corte et Lino Bianchi Barriviera font figurer le pilier, qu'ils désignent comme le " pilier de l'unité », sur les relevés qu'ils publient, ils ne disent pas si il est voilé ou pas $^{7}$. Nous pouvons toutefois supposer que c'était le cas car ces auteurs qui se sont attachés à décrire les ornements des églises et les éléments marquants qu'ils observaient, n'auraient pas manqués de remarquer les particularités des mentions portées sur ce pilier.

Dans les années 1960, Georg Gerster trouve également le pilier voilé, disant: «Depuis que le Christ, dans une vision, a soi-disant fait toucher de sa main au roi

4 Par exemple Lepage, 1999 : 965-966.

5 Alvares, $1558: 140$. Nous employons ici l'édition française du récit de voyage écrit en portugais par Francisco Alvares et publié pour la première fois en 1540. Nous avons conservé le vieux français employé par le traducteur parce qu'il rend bien compte des difficultés de la langue pour décrire le site de Lālibalā et des problèmes de compréhension que nous pose aujourd'hui le portugais du XVI ${ }^{e}$ siècle. Ce dont l'édition anglaise (Beckingham \& Huntingford, 1961: 223) ne rend pas suffisamment compte : "The house or church of Our Lady is not so large as St Saviour's, but it is very well made. It has three aisles, and the centre aisle is very high, with big loops and roses very delicately carved in the same rock. Each aisle has five columns, and upon them arches and vaults, semicircular, and well made. It has besides, a high column in the cross of the transept, over which is fixed a canopy, the tracery of which looks as if it had been stamped in wax". Cette dernière partie de la phrase ne figure pas dans la version traduite par Jean Bellère. Le mot canopy a été utilisé pour traduire le portugais «charola» (Alvares, 1943, 136), or ce terme ne se trouve pas dans les dictionnaires. Une recherche plus approfondie dans les manuscrits d'Alvares s'impose pour vérifier que ce mot a été correctement copié.

6 Simon, $1885: 310-313$.

7 Monti della Corte, 1940 : 51 ; Bianchi Barriviera, 1963 : 48-61, tav. 14-15. 
Lālibalā le pilier central, un voile le dérobe aux regards profanes. Les prêtres (...) racontent que le passé et l'avenir du monde sont écrits là. En tâtant le pilier, on n'y découvre aucune inscription dont on puisse faire état. D'ailleurs il serait naif de s'attendre à ce qu'une inscription sur le pilier livre à tout venant le principe et le sens de toute l'affaire, la solution de cette énigme indéchiffrable qu'est Lālibalā $»^{8}$.

D’après Sergew Hable Sellassie, le pilier - dont on soupçonnait qu'il portait des inscriptions - fut dévoilé en 1966 afin de le photographier en totalité. Mais l'auteur ne parvint pas à voir les photographies réalisées à ce moment-là9. C'est précisément en 1966 que Sandro Angelini fit sa première visite à Lālibalā afin d'évaluer le travail de restauration des églises pour le compte du World Monument Fund. Mais rien dans les archives de l'architecte italien, déposées à la bibliothèque municipale A. Mai de Bergame, ne permet de penser qu'il aurait vu le pilier dévoilé. Toutes les photographies prises lors des restaurations des peintures de l'église de Bēta Māryām montrent que le pilier est toujours resté couvert pendant les travaux.

Seul Stuart Munro Hay a eu le privilège de voir une photocopie de l'une des photographies faites en 1966 et de pouvoir la publier dans un ouvrage destiné au grand public puisque c'est un guide de voyage ${ }^{10}$. Il s'agit du seul document connu permettant d'attester la présence d'une inscription sur l'ámda berhān. Le texte est incomplet, mais sa teneur est suffisamment novatrice pour mériter une publication (Fig. 1) :

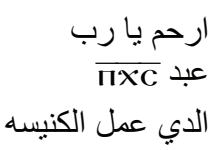

"Ô Seigneur, aie pitié

Du Serviteur du Christ qui a fait l'église »

L'inscription du pilier de Bēta Māryām est donc rédigée en arabe avec deux mots de copte, pi-christos, pour désigner l'article et l'abréviation du mot « Christ » ${ }^{11}$.

Cette inscription fournit une information de tout premier plan quant à son rédacteur: selon toute vraisemblance, il s'agit d'un chrétien d'Égypte. Sa présence en ces lieux n'est pas surprenante : l'évêque à la tête de l'Église éthiopienne, nommé par le patriarche d'Alexandrie, était un moine égyptien. Il n'était pas forcément le seul copte dans le royaume d'Éthiopie. Ainsi, au cours du règne de Lālibalā, sait-on que le métropolite Yeshāa nommé en 1210 pour remplacer Mikāèl, chassé d'Éthiopie, était accompagné de son frère Joseph. Tous deux étaient auparavant

8 Gerster, $1968: 96$.

9 Sergew Hable Sellassie, 1972 : 278 note 80.

10 Munro-Hay, 2002 : 207. Stuart Munro Hay (2002: 208-209) a tenté une traduction mais ce qu’il propose est peu clair et au final on ne sait pas bien ce que l'on doit retenir comme texte. Le texte arabe est en fait très facilement lisible et ne pose aucun problème d'interprétation.

11 Peut-être s'agit-il de l'inscription grecque qu'Antoine d'Abbadie (1859: 154-155) dit avoir vue dans une église de Lālibalā, confondant alors le copte et le grec ? Cela signifierait alors que le pilier était découvert à ce moment-là. 
Claire Bosc-Tiessé et al.

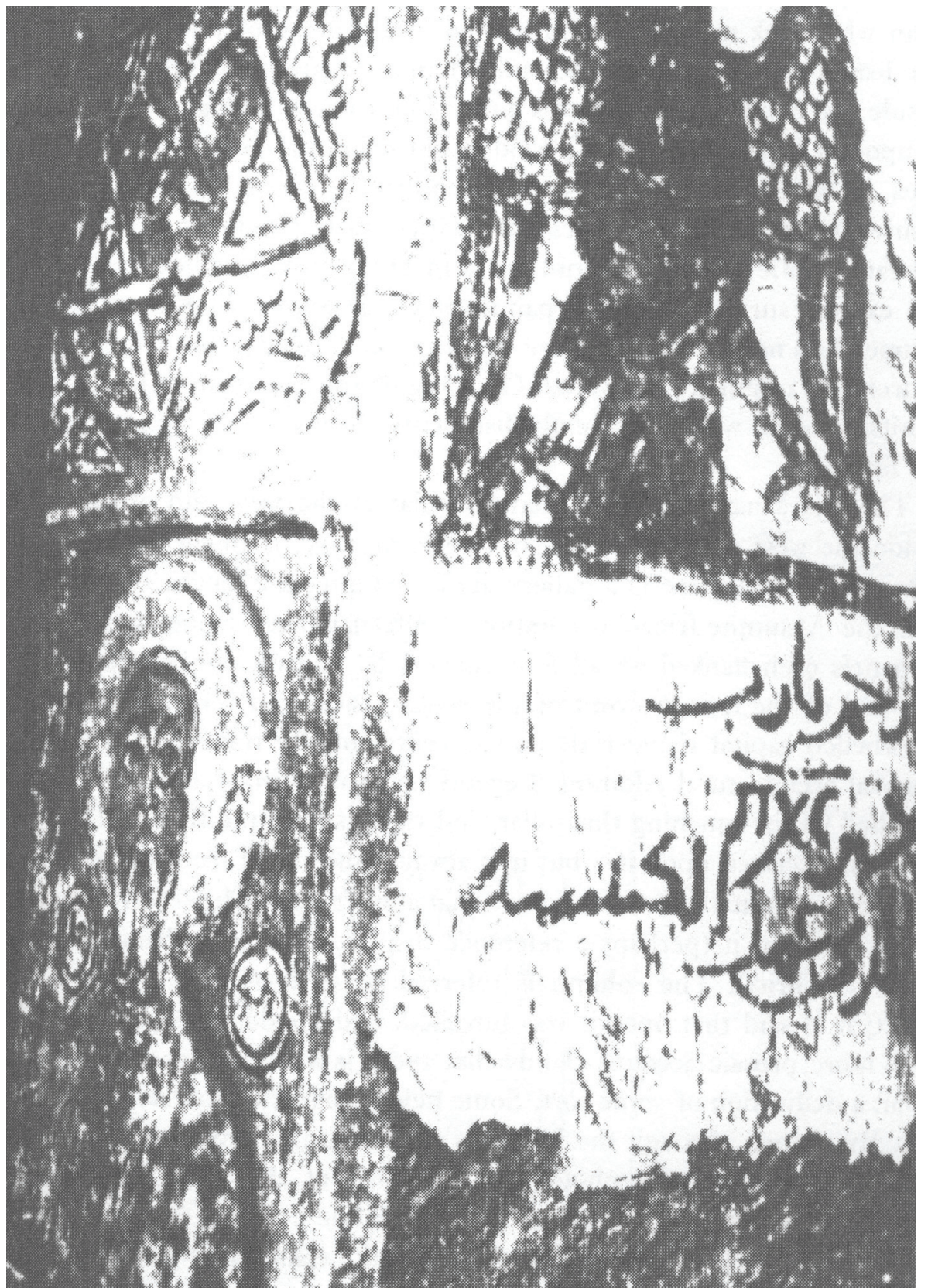

Fig. 1 - Le pilier de Bēta Māryām dévoilé d’après la photocopie publiée par Munro-Hay (2002 : 207) 
moines au monastère de Saint-Antoine ${ }^{12}$. De même, l'évangéliaire préservé à Bēta Madhanē ${ }^{c}$ Alam à Lālibalā conserve sur l'un des folios vierges, des notes en copte et en arabe qui sont des donations de terres inscrites par ou à la demande du métropolite Bartalomēwos, qui exerça sa charge durant le règne de Dāwit (1379/80$1412)^{13}$. Par conséquent, au moins le métropolite et sa cour pouvaient trouver à séjourner à Lālibalā et laisser une trace de leur passage sur les murs des églises ou sur les folios vierges des manuscrits.

La dernière ligne de l'inscription doit être considérée avec circonspection car, comme l'a si bien montré Paulo Fernando de Moraes Farias au sujet des inscriptions de l'Afrique de l'Ouest, si le texte de l'inscription est bien celui qui a été écrit par son auteur ou son commanditaire, sans qu'aucune intervention postérieure ne vienne transformer celui-ci, il n'en reste pas moins que les inscriptions sont le fruit d'une "construction rhétorique ", où l'on peut vouloir communiquer autre chose qu'un simple fait historique ${ }^{14}$. Que signifie " faire l'église » dans ce contexte? S'agit-il de celui qui a commandité l'ouvrage ? Dans ce cas, nous aurions sans doute « qui a fait faire l'église ». S'agit-il du chef de chantier ou simplement d'un artisan qui est venu travailler sur le chantier de l'église ? Mais qu'a-t-il fait ? A t-il contribué à l'excavation proprement dite ? Le fait que l'inscription se trouve précisément sur le pilier central de l'église, qui pourrait bien avoir entre autres fonctions celle d'affichage, peut conduire à penser que c'est bien de l'excavation qu'il s'agit. C'est en cela que cette inscription relève de la catégorie "texte de fondation », catégorie qui rassemble des textes souvent formulés comme des prières mais qui servent aussi à indiquer l'action d'un donateur, d'un fondateur ou ici d'un constructeur.

Par ailleurs, on peut souligner aussi que la photographie publiée par Stuart Munro-Hay montre que l'inscription est elle-même peinte sur un enduit, le même que celui qui a servi pour les peintures qui se trouvent au-dessus et sur le côté de l'inscription. Cela reviendrait par conséquent à penser que l'excavation de l'église, au cours de laquelle un pilier central est réservé, et la décoration de celle-ci sont intervenues dans le même temps. Les peintures que nous apercevons sur la photocopie publiée par Stuart Munro-Hay réprésentent une étoile de David, un aigle à deux têtes ${ }^{15}$ et un saint difficile à identifier sur la photocopie. Barbu, il est entouré d'un monogramme en caractères guèzes ${ }^{16}$ pour lequel on ne peut distinguer que les deux caractères « $\boldsymbol{\varkappa} \mathbf{\Lambda}$ 》 (rus) qui terminent son nom.

Nous pouvons donc retenir deux informations. Tout d'abord, le voile du pilier de Bēta Māryām vient bien couvrir une inscription dont la teneur est suffisamment dérangeante aux yeux du clergé local. Ce qui gêne en l'occurrence ce sont les langues de l'inscription - l'arabe et le copte - parce qu'elles sont un témoignage direct d'une intervention extérieure longtemps évoquée dans la tradition, mais

\footnotetext{
12 Perruchon, 1898-1899 : 84 ; Khater \& Burmester, 1970 :91

13 Au sujet de cet évangile, voir l'article de Marie-Laure Derat sur les donations de terre du roi Lālibalā, dans ce même volume ainsi que Bosc-Tiessé \& Derat (2010).

14 Moraes Farias, 2004 : xli.

15 À l'identique de ceux peints dans les pendentifs des arches de la nef centrale.

16 Tracés dans deux cercles, disposés de part et d'autre du buste du personnage.
} 


\section{Claire Bosc-Tiessé et al.}

aujourd'hui totalement déniée ${ }^{17}$; de même que le contenu de l'inscription, à supposer que certains ecclésiastiques étaient en mesure de la lire au moment où la décision de voiler l' àmdà berhàn a été prise. Par ailleurs, cette inscription vient combler une lacune de la documentation s'agissant du moment de la fondation des églises. Nous disposons d'autels dont la dédicace renvoie très clairement au roi Lālibalā, ainsi qu'une donation de terres de ce même souverain pour l'église de Bēta Medhānē Alam, mais aucun écrit ne faisait allusion précisément au chantier des églises. L'inscription, certes lapidaire, vient combler ce manque.

En outre, l'emploi du pilier de Bēta Māryām comme média sur lequel on peut inscrire les événements importants dont il faut se souvenir peut expliquer certaines mentions que l'on trouve dans les manuscrits de la région. Par exemple, dans l'église d'Ašatan Māryām, qui surplombe le site de Lālibalā, on trouve un évangile dans lequel est inscrit un colophon datant du roi Zar'a Yā'eqob (1434-1468). Dans ce dernier, figure l'information suivante :

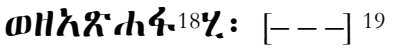

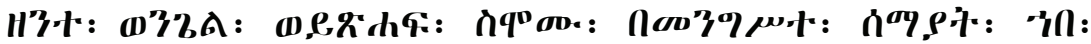

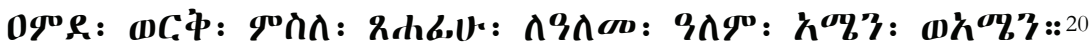

«Et celui qui a fait écrire cet évangile [- - ].

Que leurs noms soient écrits dans le royaume de cieux, sur le pilier doré, avec son scribe. Pour l'éternité, amen et amen ».

On peut rapprocher ce texte d'un autre colophon, écrit celui-ci dans le manuscrit des Nagara Māryàm conservé à Bēta Māryām, que l'on peut dater du XVIII' siècle, où l'on retrouve une mention similaire :

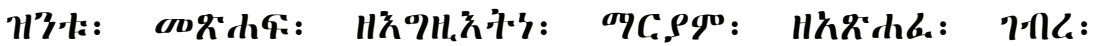

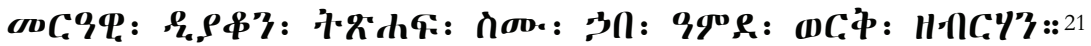

«Ce livre de Notre Dame Marie qu'a fait écrire Gabra Mar’āwi, le diacre.

Que son nom soit écrit sur le pilier doré de lumière.»

17 Alvares, $1558: 147$.

18 Sic.

19 Quatre ou cinq lignes ont été ici effacées.

20 EMML 7031, fol. 146v-147r.

21 EMML 7229, fol. 118v. 
Un texte que l'on retrouve dans différents manuscrits fait aussi allusion à cette pratique $^{22}$. Énumérant les dates de consécration des églises, il débute par les termes suivants :

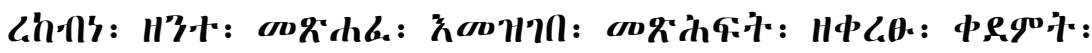

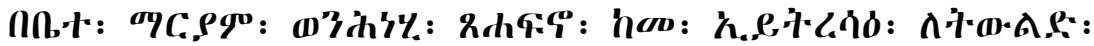 H,eov\%: : 23}

«Nous avons trouvé cet écrit dans la collection des livres que les anciens avaient gravée à Bēta Māryām, et nous l'avons copié afin qu'il ne tombe pas dans l'oubli pour les générations à venir. »

Ce passage n'est pas facile à interpréter : comment faut-il interpréter cette collection de livres? Comment pourrait-elle être gravée sur un pilier? Quoiqu'il en soit, ce n'est pas l'énumération des dédicaces des églises qui suit ce texte dans les manuscrits, que l'on trouve sur le pilier. Toutefois, ce petit texte signale bien la position particulière de l'église de Bēta Māryām à Lālibalā comme lieu de conservation de la mémoire.

\section{Conclusion}

Nous avons mis ici l'accent sur les inscriptions en arabe qui soulignent les rapports avec l'Égypte copte à travers le temps. Nombre des inscriptions guèzes sont du même ordre, invitant à se remémorer le passage d'un pèlerin et à prier pour lui. Mais elles sont le plus souvent très difficiles à lire et ne peuvent pas être replacées dans un contexte historique particulier.

En ce qui concerne les inscriptions arabes et coptes, en revanche, ce sont les liens avec l'Égypte qui sont ainsi rappelés. Lorsque Francisco Alvares visite les églises de Lālibalā peu après 1520, les informations orales qu'il recueille au sujet du site sont les suivantes: "cette superbe structure de ces églises fut dressée par Gibetes, c'ét à dire hommes blans : car les habitans méme de là, connaissent bien leur portée ne pouvoir ataindre à telle perfection $»^{24}$. Dans l'édition et la traduction anglaise, on trouve une information qui est absente du français : «They told me that all the work on these churches was done in twenty-four years, and that this is written, and that they were made by Gibetas (...) They say that King Lalibela ordered this to be done $»^{25}$. Au XVI siècle, à Lālibalā, il y a donc bien l'idée d'une

\footnotetext{
22 La plus ancienne version que nous connaissons aujourd'hui se trouve dans un livre d'Évangiles du XVe siècle de Bēta Golgotā (mf. EMML 6934, fol. 163v). Il a ensuite été utilisé comme colophon dans deux copies (en l'état actuel de nos connaissances) des Actes de Lälibalä (le manuscrit Cerulli 223 cité ci-après et le mf. EMML 6964, fol. 132rv, daté de 1694-1695). Sergew Hable Sellassie (1972 : 272 note 52) faisait référence à un autre exemplaire de ces Actes conservés à Bēta Gabre’ēl. Ce manuscrit ne figure pas parmi les microfilms réalisés par le projet EMML.

23 D'après le manuscrit Cerulli 223, fol. 124v. Sergew Hable Sellassie (1972: 272 note 52) donnait seulement une version anglaise du texte vu dans les Actes de Lalibela de Bēta Gabre'ēl : «We recorded this document from the inscription engraved in Bete Mariam by the old generation ». L'ayant lu, Claude Lepage (1999: 966) parle de ce texte qu'il n'a pas vu en disant qu'il a été copié sur une inscription gravée sur le pilier de Bēta Māryām.

24 Alvares, 1558 : 147 ; Alvares, 1943, 140.

25 Beckingham \& Huntingford, $1961: 227$.
} 


\section{Claire Bosc-Tiessé et al.}

participation égyptienne en ce qui concerne la construction - comme l'indique l'inscription de Bēta Māryām - mais rien qui puisse induire un patronage égyptien ${ }^{26}$.

\section{Bibliographie}

Abbadie A. (d'), 1859, Catalogue raisonné des manuscrits éthiopiens appartenant à Antoine d'Abbadie, Paris, Imprimerie Nationale.

Alvares F. ,1558, Historiale description de l'Éthiopie contenant vraye relation des terres, \&o païs du grand Roy, \& Empereur Prete Ian, l'assiette de ses royaumes et provinces, leurs coutumes, loix, \& religion, avec les pourtraits de leur temples \& autres singularitez, cy devant non cogneues, trad. Jean Bellère, Anvers.

Alvares F., 1943, Verdadeira informação das terras do Preste João das Índias pelo Padre Francisco Álvares, Divisão de publicações e biblioteca, Agéncia geral das colónias.

Beckingham C. F. \& Huntingford G. W. B. (1961), The Prester John of the Indies. A true relation of the lands of the Prester John being the narrative of the Portuguese Embassy to Ethiopia in 1520 written by Francisco Alvares, Cambridge, The Hakluyt Society.

Bianchi Barriviera L., Le Chiese in roccia di Lalibelà e e di altri luoghi del Lasta, Roma, Istituto per l'Oriente, 1963, (Estratto da «Rassegna di Studi Etiopici », volumi XVIII e XIX, anni 1962 e 1963).

Bosc-Tiessé C., Derat M.-L., 2010 (sous presse), “Acts of writing and authority in $\mathrm{Beg}^{\mathrm{w}}$ ena and Lasta between the $15^{\text {th }}$ and $18^{\text {th }}$ century: a regional administration comes to light", Journal of Northeast African Studies.

Gerster G., 1968, L'Art éthiopien. Églises rupestres, La Pierre-qui-Vire, Zodiaque.

Khater A., Burmester O. H. E., 1970, History of the Patriarchs of the Egyptian church, known as the History of the Holy Church by Sawirus ibn al-Mukaffa, Bishop of alAsmunin, Le Caire.

Lepage C., 1999 (juillet-octobre), Les peintures murales de l'église Betä Maryam à Lalibala, Éthiopie (rapport préliminaire), Comptes rendus des séances de l'année de l'Académie des inscriptions et belles-lettres, 3, p. 901-966.

Lepage C., 2002 (ajnvier-mars), Un métropolite égyptien bâtisseur à Lalibäla (Éthiopie) entre 1205 et 1210, Comptes rendus des séances de l'année de l'Académie des inscriptions et belles-lettres, 1, p. 141-174.

Monti della Corte A. A., 1940, Lalibelà. Le chiese ipogee e monolitiche e gli altri monumenti medievali dei Lasta, fotografie rilievi del centurione Elio Zacchia, disegni originali di Lino Bianchi Barriviera, Roma, Società italiana arti grafiche.

Moraes Farias P. F., 2004, Arabic Medieval Inscriptions from the Republic of Mali. Epigraphy, Chronicles and Songhay-Tuareg History, Oxford, Oxford University Press.

Munro-Hay S., 2002, Ethiopia, the Unknown Land: a Cultural and Historical Guide, London, Tauris.

${ }^{26}$ Contrairement à l'hypothèse de Claude Lepage, 2002 : 141, 170. 
Perruchon J., 1898-1899, Notes pour l'histoire d'Éthiopie. Extrait de la vie d'abba Jean, 74 e patriarche d'Alexandrie, relatif à l'Abyssinie (texte arabe et traduction), Revue Sémitique 6, p. 267-271 ; 7, p. 76-88.

Sergew Hable Sellassie, 1972, Ancient and Medieval Ethiopian History to 1270, Addis Ababa, United Printers.

Simon G., 1885, Voyage en Abyssinie et chez les Gallas-Raias. L'Éthiopie, ses moeurs, ses traditions, le négouss Iohannès, les églises monolithes de Lalibéla, Paris.

Verdadeira informação das terras do Preste João das Índias pelo Padre Francisco Álvares, 1943, Divisão de publicações e bibloteca, Agéncia geral das colónias.

\section{Résumé / Abstract}

Bosc-Tiessé C., Derat M.-L., Fritsch E., Wadi Awad Abullif, 2010, Les inscriptions arabes, coptes et guèzes de Lālibalā, Annales d'Éthiopie, 25, 43-53.

Cet article publie et traduit des inscriptions arabes, coptes et guèzes relevées dans trois églises du site de Lālibalā : à Bēta Māryām, Bēta Giyorgis et Bēta Libānos. Pour la plupart, ce sont de simples ex-voto. Une attention particulière est accordée à l'une des inscriptions, figurant sur le pilier central de l'église de Bēta Māryām (l'āmda berhān) qui fait allusion à la construction de l'église.

Mots-clefs : Lālibalā, inscriptions, arabe, copte, guèze, ex-voto.

The Arabic, Coptic and Ge'ez inscriptions in Lālibalā - In this article, we edit and translate Arabic, Coptic and Ge'ez inscriptions from three churches of the Lālibalā's site: Bēta Māryām, Bēta Giyorgis and Bēta Libānos. Most of them are simple ex-voto. A particular attention is given to one of these inscriptions, placed on the central pilar of the Bēta Māryām church ('āmda berhān) which refers to the carving of the church.

Keywords: Lālibalā, inscriptions, arabic, coptic, ethiopic, ex-voto. 